\title{
82
}

$x^{2} / 5$

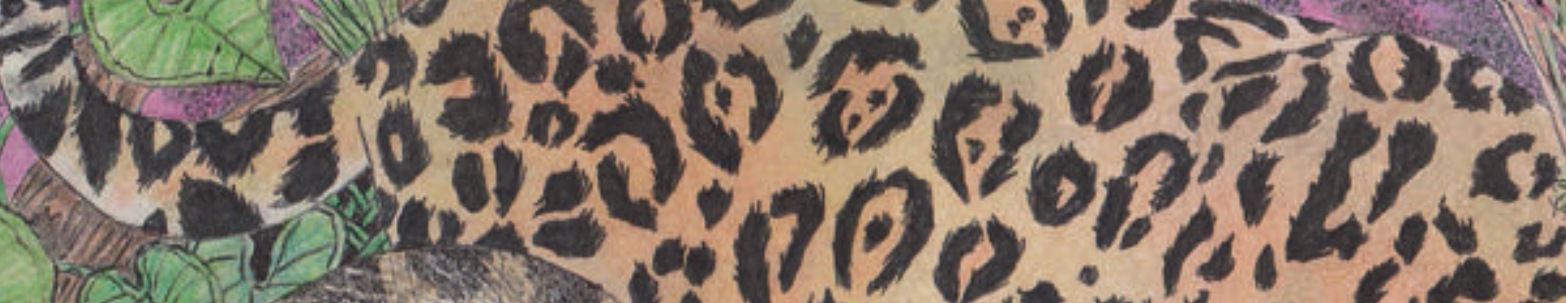






\section{LA EXPERIMENTACIÓN EN LAS CIENCIAS NATURALES Y SU IMPORTANCIA EN LA FORMACIÓN DE LOS ESTUDIANTES DE BÁSICA PRIMARIA}

\section{Experimentation in the Natural Sciences and its Importance in the Training of Primary School Students}

\section{Experimentação em ciências naturais e sua importância na formação de alunos do ensino fundamental}

\section{Aura Ximena García Viviescas* Yineth Alejandra Moreno Sacristán**}

Fecha de recepción: 25 de mayo de 2019

Fecha de aprobación: 30 de noviembre de 2019

\section{Resumen}

En este artículo se reflexiona sobre la importancia de la experimentación en la enseñanza de las ciencias naturales, a través del análisis de los planteamientos de diferentes autores. Dicha reflexión exterioriza la necesidad de implementar prácticas experimentales en el aula, especialmente en el nivel de educación básica, donde las habilidades de curiosidad y observación se configuran como un elemento clave en la articulación de lo biológico y lo social. En este proceso subyace la observación detallada del fenómeno, la construcción de preguntas, el planteamiento de una hipótesis, su comprobación y la "artifilización" del objeto de estudio, acompañada de los propósitos del maestro en la enseñanza. Por lo anterior, la experimentación se configura como un mecanismo por el cual el estudiante se convierte en el protagonista de su aprendizaje y sujeto consciente de las relaciones que él establece con su entorno.

Palabras clave: aprendizaje; conocimiento; curiosidad; experimentación; observación

\footnotetext{
* Estudiante de la Maestría en Docencia de las Ciencias Naturales, Universidad Pedagógica Nacional; Magíster en Intervención Social en las Sociedades del Conocimiento, Universidad Internacional de la Rioja; Licenciada en Biología, Universidad Pedagógica Nacional (Bogotá, Colombia). Orcid: https://orcid.org/0000-0003-0021-7827. Correo electrónico: axgarciava@gmail.com

* Estudiante de la Maestría en Docencia de las Ciencias Naturales, Universidad Pedagógica Nacional; Licenciada en Biología, Universidad Pedagógica Nacional (Bogotá, Colombia). Orcid: https://orcid.org/0000-0002-2473-918X. Correo electrónico: yamorenos@upn.edu.co
} 


\section{Abstract}

This article reflects on the importance of experimentation in the teaching of natural sciences, through the analysis of the approaches of different authors. This reflection externalizes the need to implement experimental practices in the classroom, especially at the basic education level, where curiosity and observation skills are configured as a key element in the articulation of the biological and the social. Underlying this process is the detailed observation of the phenomenon, the construction of questions, the formulation of a hypothesis, its verification and the "artifilization" of the object of study, accompanied by the teacher's aims in teaching. Therefore, experimentation is configured as a mechanism by which the student becomes the protagonist of his learning and a conscious subject of the relationships he establishes with his environment.

Keywords: curiosity; experimentation; knowledge; learning; observation

\section{Resumo}

Este artigo reflete sobre a importância da experimentação no ensino de ciências naturais, através da análise das abordagens de diferentes autores. Essa reflexão externaliza a necessidade de implementar práticas experimentais em sala de aula, principalmente no ensino fundamental, onde as habilidades de curiosidade e observação são configuradas como um elemento-chave na articulação do biológico e do social. Subjacente a esse processo está a observação detalhada do fenômeno, a construção de questões, a formulação de uma hipótese, a sua verificação e a "artifilização" do objeto de estudo, acompanhada dos objetivos do professor no ensino. Portanto, a experimentação é configurada como um mecanismo pelo qual o aluno se torna protagonista de seu aprendizado e sujeito consciente das relações que estabelece com seu ambiente.

Palavras chave: aprendizagem; conhecimento; curiosidade; experimentação; observação 
Hacer de la experimentación una plataforma de conocimiento contribuye a un camino en la imagen de la ciencia. La manera de presenta los experimentos no debe ser solamente descriptiva o narrativa para reforzar el papel de las teorías, sino que debe avanzar hacia la caracterización de experimentos que involucren problemáticas y que tengan una riqueza conceptual en sí mismos (vida propia), crear necesidades donde el experimento 'hable' y se comunique, crear situaciones específicas donde la naturaleza se 'despliegue' y muestre comportamientos, esto es, hacer de la experimentación una actividad humana en el sentido de Hacking.

(GARCÍA Y ESTANY, 2010)

\section{Introducción}

La educación en el siglo xx se enfrenta a nuevos desafíos, fruto de una sociedad en constante cambio y transformación, por lo cual, la escuela ha de formar sujetos capaces de comprendery dar explicaciones a situaciones problema en su contexto; por supuesto, esto requiere de una transformación radical en la forma tradicional de enseñar Ciencias Naturales, donde evidentemente hay una prevalecía sobre desarrollo de contenidos, conceptos y terminologías por encima de las practicas experienciales. En este sentido, la educación en Ciencias debe fomentar en los niños y jóvenes "la posibilidad de vivir en carne propia el proceso mismo de investigar el mundo" (Furman, 2016, p. 32).

En particular, el conocimiento científico se construye a partir de la experimentación, el cual "es un proceso que lleva a la comprobación de fenómenos naturales a través de diferentes técnicas y procedimientos, transformándolos en principios o leyes a lo largo de los siglos" (Canizales,et ál., 2004, p. 26); de tal forma que implementar prácticas experimentales en el aula es un gran reto para los maestros, porque implica aproximar a los estudiantes a la forma como se consolida dicho conocimiento.

En primer lugar, la experimentación trasciende de la observación, porque ofrece la posibilidad de estudiar los fenómenos naturales con mayor exactitud, profundidad y significación; además, posibilita la manipulación de variables y el estudio del fenómeno a partir del análisis de datos y el comportamiento de este, mientras que la observación "estudia el curso natural de las cosas [...] en este caso no se alteran las condiciones naturales del fenómeno" (Carvajal, 2011, p. 46). En segundo lugar, las clases de Ciencias Naturales en la escuela primaria deben favorecer que los niños y jóvenes expongan y discutan sus explicaciones respecto a su entorno. En tercer lugar, el alumno debe pasar de la aceptación a la construcción de conocimientos científicos a partir de la comprensión de lo natural y así poder intervenir en ella.
Por último, la experimentación como camino a la problematización de fenómenos concretos de la naturaleza, sin importar que las actividades experimentales que se realicen para la enseñanza de las Ciencias Naturales tengan diferentes objetivos y alcances - todos de manera subsecuente-, parten de la curiosidad como parte fundamental de la experimentación, porque permite explorar un fenómeno natural; formular preguntas, tratar de resolverlas, confrontar los resultados que permitan al estudiante desarrollar un proceso de interpretación y construir nuevas explicaciones a los problemas cotidianos y, al final, generar nuevas preguntas, que serán un elemento clave para seguir construyendo conocimiento científico en el aula y, en suma, contribuir en la resolución de situaciones problema a partir de actividades de experimentación por parte de los estudiantes.

\section{La relevancia de la experimentación en la enseñanza de las Ciencias Naturales}

En primera instancia, se precisa acerca del papel de la observación en la experimentación escolar. La observación se entiende como la experiencia y el conocimiento del mundo sensible que posibilita el establecimiento de relaciones e interpretaciones del fenómeno natural estudiado, esto requiere de la variación de las causas o determinante del objeto de estudio, es así como se constituye la experimentación (Repositorio Educación, 2012). Sin ir más lejos, cuando un estudiante aprende acerca de la caída de los cuerpos en un plano inclinado y desarrolla una observación activa en el estudio del fenómeno inicia un proceso de experimentación, porque incidentalmente varía las condiciones de los cuerpos para comprender su naturaleza.

En efecto, la experimentación en el estudio de las ciencias es la base estructurante de los fenómenos naturales. Para explicarlo, se tomará como referente los aportes de García (2009), para el cual "construir experiencia es llenar de sentido una actividad en la que la práctica es un medio de constante reflexión sobre el fenómeno abordado" (p. 34); es así como el experimento se consolida como una estrategia indispensable para estudiar los fenómenos naturales en respuesta a los intereses del investigador, ya que esto aporta seriedad, validez y rigurosidad en la comprobación de sus hipótesis. En efecto, los científicos han podido dar respuestas a fenómenos naturales que antes sería casi imposible explicar a través de la experimentación.

En la Edad Media ya se reconocía de forma generalizada la importancia de los experimentos, pero solo hasta la segunda mitad del siglo xvı Galileo Galilei busca una planificación para su desarrollo [...] al crear unos procedimientos que lo encaminan a resolver varias de sus hipótesis. (Tamayo, 2004) 
Sin duda, para Galileo la experimentación constituía el eje central del método científico, partiendo de un enfoque teórico en el planteamiento de la técnica y en la elaboración de datos experimentales que apoyaron los descubrimientos de tan ilustre personaje.

En definitiva, el experimento tal cual como hoy se conoce es "un proceso riguroso, constante, planificado y de confrontación necesario para la construcción de conocimiento científico" (Tamayo, 2004, p. 76), así es como, el experimento ha ganado status a lo largo de los siglos y se constituye como un mecanismo veraz en las dinámicas de la ciencia.

La experimentación cobra gran relevancia en la comprensión del mundo circundante de forma clara y veraz, posibilitando la construcción de conocimiento científico; porque implica

utilizar mecanismos que aíslan el fenómeno en estudio, reproducir muchas veces el curso del proceso en condiciones fijadas y sometidas a control para finalmente de forma planificada, variary, buscar diferentes combinaciones con el objetivo de obtener el resultado buscado. (Tamayo, 2004, p. 24)

A partir de lo anterior, es válido afirmar que las Ciencias Naturales avanzan en la medida en que se generan nuevos conocimientos a partir de la experimentación constante y rigurosa de un fenómeno en particular. Por otro lado, posibilita el ir más allá de la observación, porque examina con detalle el fenómeno o situación problema evidenciada en la naturaleza y también construye una interpretación y explicación sólida acerca de lo observado.

En conjunto, se puede decir que la experimentación es indispensable en la enseñanza de las Ciencias Naturales, por cuanto vincula la observación y el experimento para constituir explicaciones sobre un sistema de hechos visibilizados en un fenómeno natural. Aunque los fines de la observación radican en estudiar el curso natural del fenómeno y el experimento en la manipulación de las variables, los dos convergen en un mismo fin: la comprensión de la naturaleza.

Al mismo tiempo, la experimentación, al permitir el análisis detallado de los hechos y el acercamiento al conocimiento científico, vislumbra en los aprendices las condiciones naturales del fenómeno, las consecuencias de su alteración e impacto sobre el mismo, además de la formulación de soluciones coherentes con la realidad de sus contextos; es así como a partir de este mecanismo, los estudiantes identifican con mayor claridad la aplicación de las ciencias en la resolución de problemas cotidianos.
En otras palabras, lograr que el experimento adquiera un valor significativo que promueva situaciones, que permita explorar, que genere una estrecha relación entre el experimento y el experimentador, debe ser uno de los objetivos primordiales de este tipo de estrategias en el aula. Pero la noción que se tiene del experimento tiene otro valor que fue adquirido con la corriente positivista, su papel ha sido comprobador, verificador o falseador de teorías, el cual ha permanecido por muchos años, reduciendo así la importancia del experimento en la construcción del conocimiento.

Para explicarlo claramente, la función de la experimentación en la enseñanza de las Ciencias Naturales no tiene como fin comprobar la teoría o eliminar las ideas de la mente, sino más bien una evaluación constante de las explicaciones generadas, buscando coherencia entre la idea expresada y los hechos (Adúriz et ál, 2011); para ello consideramos que los estudiantes deben partir de un análisis de los fenómenos naturales a estudiar, lo que implica a su vez una observación detallada, el registro de datos, la lectura y contraste del conocimiento propio con el de otros autores, la comprensión de su contexto, el planteamiento de explicaciones y por último la búsqueda de soluciones al problema explorado. Estos elementos en conjunto cobran sentido de la experimentación en el aula, al brindar herramientas a los niños y jóvenes, para ensamblar el conocimiento científico escolar en vía de actitudes científicas que evidencien impacto positivo frente a los procesos de formación en ciencias en esta etapa del desarrollo escolar.

\section{La experimentación en la escuela y su papel en la educación primaria}

En este apartado se plantean los interrogantes: ¿cuál es la importancia de la experimentación en la enseñanza de las Ciencias Naturales para el nivel de básica primaria? y ¿por qué es importante la experimentación para comprender la causa de los fenómenos naturales abordados en la educación básica?; con el propósito de dar respuesta a las preguntas anteriores, es necesario esbozar la necesidad de abordar las formas como se enseñan las Ciencias Naturales y las posibilidades que ofrece la experimentación en el aula.

La enseñanza de las Ciencias Naturales que se ofrece en "la mayoría de los casos se trata de una enseñanza rígida, exhaustiva y repetitiva, en lugar de creativa e inventiva" (Rodríguez, 2007, p.7); lo anterior puede surgir como respuesta a un proceso de enseñanza mecánico, donde los fenómenos naturales son abordados desde lo teoría y poco desde la práctica, dejando de lado en muchas ocasiones, la experimentación y con ello el desarrollo de destrezas procedimentales necesarias para la comprensión y abordaje de las Ciencias Naturales. 
Las repercusiones de la casi nula experimentación traen como consecuencia que los estudiantes, aunque repitan un contenido, pocas veces puedan construir su propio conocimiento, obligándolos a cimentar un concepto errado de las ciencias y provocando la asimilación de conductas memorísticas que dificultan la interpretación de los fenómenos estudiados en el aula, la poca formulación de preguntas en torno a su contexto y, por consiguiente, las posibles respuestas desde las ciencias.

En contraste con el desarrollo de prácticas de tipo memorístico, hay maestros que desarrollan en sus clases actividades que fomentan destrezas científicas, es así como "las clases de Ciencias Naturales deben ser un espacio para que los niños y jóvenes expongan y discutan sus preguntas y explicaciones respecto a lo que ocurre en su contexto" (Oñate, 2016, p. 28). Una clase de Ciencias Naturales que propicie la duda y el cuestionamiento construye el sendero hacia la búsqueda de explicaciones que impulsen la relación del niño con el medio natural de manera armónica y responsable. De igual forma, puede incentivar en los estudiantes interés por la búsqueda de explicaciones de su contexto y, seguramente, esto llevará a la construcción de conocimiento.

Desde los planteamientos señalados, el valor educativo del estudio de las Ciencias Naturales no radica en un aprendizaje memorístico, sino cuestionable y capaz de transformar la realidad de los estudiantes, porque "...pocas experiencias pueden ser estimulantes para el desarrollo de las capacidades intelectual y afectivas de los estudiantes, como el contacto con el mundo natural y el despliegue de sus potencialidades para conocerlo..." (Canizales, et ál., 2004, p.9); las cuales parten de una observación superficial de la naturaleza y posteriormente van generando mayor interés y curiosidad en los niños y jóvenes.

En el nivel de educación primaria se pretende que los estudiantes, según Mora, et ál. (2002)

desarrollen la capacidad de pensar y resolver problemas [...] desarrollen en los niños el potencial creador [...] promover en los niños el trabajo de aprender haciendo para descubrir, redescubriry así construir el conocimiento [...] promover el desarrollo de una actitud científica en los niños [...] facilitar la comprensión de conceptos científicos, principios y fundamentos teóricos de las Ciencias Naturales, con la finalidad de que el niño sea capaz de comprender los fenómenos naturales del entorno que afectan directamente su vida cotidiana. (p.21)

En consecuencia, las Ciencias Naturales deben propiciar la construcción de conocimientos, y estimular en los estudiantes la capacidad de aprender de forma autónoma, dejando de ser un agente pasivo para convertirse en uno activo; sujeto capaz de modificary transformar su entorno positivamente para su mejor desarrollo dentro de una sociedad determinada.

La modificación y transformación del entorno por parte de los estudiantes, implica que las Ciencias Naturales sean abordadas en el aula como un conocimiento que interpela la realidad y que constantemente está en construcción. En particular, cuando el maestro diseña e implementa una metodología basada en procesos de experimentación, es probable que se facilite el aprendizaje de los contenidos a través de su problematización; entonces el docente acompaña al estudiante en la construcción de su propio conocimiento, a partir de la observación activa del contexto donde están inmersos.

Hoy, la perspectiva a la que se da lugar cuando se habla de la enseñanza de las Ciencias Naturales consta no solo de "la comprensión y uso de conceptos, principios, leyes, modelos y teorías" (Oñate, 2016, p.6); sino también, de "que los estudiantes aprendan ciencias, a partir de la utilización de la experimentación como el principal camino que les permite adquirir conocimientos de una manera más sólida" (Pedrinaci, 2012, p.7).

Por lo anterior, es necesario precisar la diferencia entre experimento científico y trabajo práctico experimental, ya que los objetivos de cada uno son diferentes; por un lado, el experimento científico: tiene como propósito consolidar conocimiento científico. Mientras que el trabajo práctico experimental: busca generar un proceso de comprensión e interpretación de los fenómenos naturales. El experimento científico y el trabajo práctico experimental se encuentran directamente relacionados con la experimentación y la construcción de conocimiento (Canizales et ál., 2004).

En contraste con lo anterior, revalorando la importancia que tiene el experimento en la constitución y el desarrollo científico, recientes estudios históricos y filosóficos de las ciencias han resaltado una visión integral de la actividad científica a través de la cual se asume que la experimentación y la teorización, no solo son dimensiones equiparables (Hacking, 1996; Ordóñez, et ál., 2002) sino también interdependientes, por lo cual es necesario hacer una reflexión sistemática sobre el rol que tiene la actividad experimental en la construcción del conocimiento, no solo científico sino en su enseñanza en la escuela.

En relación con el experimento científico, este es aquel que "involucra la manipulación intencionada de una acción para analizar sus posibles afectos dentro de una situación artificial” (Tamayo, 2004) 
además de ser un estudio en el que se controla y se manipula deliberadamente una o más variables independientes que inciden en un fenómeno para analizar las consecuencias de esa manipulación sobre una o más variables dependientes dentro de una situación de control para el investigador. (Baptista et ál., 1994, p. 80)

Contrario al experimento científico, el trabajo práctico experimental se refiere a las actividades de enseñanza de las Ciencias Naturales en las que los estudiantes han de utilizar determinados procedimientos para reproducir un fenómeno o analizar una parte del mundo a estudiar (Leite, 2001). Estos trabajos prácticos experimentales implican la articulación de diferentes tipos de actividades, mediante un enfoque integrado en el que la teoría y la práctica se tejen de manera simultánea (Del Carmen, 2010).

A fin de subrayar la experimentación investigadora, tiene por condición la variación de las condiciones del objeto de estudio, para aprehender relaciones o dependenciasy, de esta suerte, comprobar o rechazar hipótesis; mientras, que la experimentación escolar se realiza con fines didácticos, para consolidar conocimiento y formar en Ciencias Naturales. (Repositorio Educación, 2012).

Eventualmente, el experimento escolar se realiza con dos propósitos: el comprobatorio y asertorio, este sucede con posterioridad a la explicación, distinto del experimento con base en inferencias que sucede previo a la explicación, donde el docente y el estudiante intercambian ideas e interactúan en el análisis y problematización de los fenómenos naturales, en búsqueda de alternativas de solución a las mismas.

Luego de clarificar las diferencias entre el experimento de tipo investigativo y escolar, es posible afirmar que la experimentación en la enseñanza de las Ciencias Naturales cobra importancia, ya que es "una estrategia práctica donde el alumno pone en juego los conocimientos adquiridos, además de permitirle explorar, observar, analizar, concluir y crear sus propias hipótesis, desarrollando así habilidades relacionadas con el pensamiento analítico, crítico, creativo y reflexivo" (Aragón, 2011, p.7); en otras palabras, una ciencia contextualizada capaz de darle sentido, significado y explicación a la realidad de los estudiantes.

Brevemente, la experimentación en la enseñanza de las Ciencias Naturales faculta a los estudiantes a desarrollar habilidades científicas como la de describir los hechos observados, argumentar, socializar y crear nuevos conocimientos en los cuales los estudiantes contrasten el marco de referencia propio con los autores y el análisis crítico de los mismos. Estas actividades permitirían la consolidación y construcción de nuevos conocimientos y la aplicación de lo aprendido. Por ejemplo, como cuando se ponen de manifiesto las leyes del movimiento al aplicarle una fuerza a una silla para cambiarla de posición generando movimiento y cambiando su estado de inercia, la explicación de este fenómeno dentro de un sistema de ideas sencillas consolidaría el conocimiento escolar y práctico del estudiante.

\section{Propósitos y alcances de la actividad experimental}

La actividad experimental es fundamental en la enseñanza de las Ciencias Naturales, da la adquisición de herramientas primordiales para la construcción del conocimiento científico en los estudiantes; la experimentación en el aula tiene como propósito principal favorecer la comprensión y apropiación del conocimiento científico, lo cual se ha convertido en uno de los mayores retos contemporáneos de los maestros. Una de las metas fundamentales para la formación en Ciencias Naturales en la escuela, consiste en procurar que los estudiantes se aproximen progresivamente al conocimiento científico, tomando como punto de partida su conocimiento natural del mundo y fomentando en ellos una postura crítica que responda a un proceso de análisis y de reflexión continua y cada vez de mayor complejidad (Peña, 2012).

Lo anterior involucra al maestro en tanto, planifica e implementa rigurosamente actividades experimentales en el aula que vinculan la exploración, la observación activa e interacción de los estudiantes con su entorno cotidiano mediante situaciones y experiencias de fácil ejecución; con certeza, los aprendices, desarrollan una mejor competencia en la interpretación y comprensión de los fenómenos naturales y los procesos involucrados en su estudio, así pues, potenciando el desarrollo del pensamiento científico escolar.

Por otra parte, es necesario señalar que los maestros y los estudiantes tienen acceso a otros medios de información, no solo en el plan formal que se imparte en la escuela, sino a través de medios como la televisión, los vídeos, las revistas, el internet, por lo que poseen mayores elementos para poder dialogar, argumentar y discutir acerca de los fenómenos naturales y de los adelantos científicos que se están generando en la actualidad (Alegría, 2013), pero que implican un escenario capaz de discutir y debatir lo cotidiano con los conocimientos escolares. Ante esta transformación de los actores educativos, los profesores de educación básica (preescolar, primaria y secundaria) deben poseer conocimientos relacionados con la ciencia y desarrollar estrategias metodológicas y didácticas para promover en el alumno la adquisición de habilidades y destrezas que les permitan comprender e interrogar su 
entorno, ya que esta curiosidad e interés científico les ayudará a comprender el mundo natural. Dada la problemática y el reto de la enseñanza-aprendizaje de las Ciencias Naturales, se hace cada vez más necesario propiciar diferentes estrategias didácticas para relacionar las experiencias de la vida cotidiana con las temáticas propias de los currículos, con el fin de facilitar dicho proceso y en el cual los trabajos experimentales cobran gran relevancia.

Tomando como referente a Caamaño (2003), se proponen cuatro tipos de trabajos experimentales que se llevan a cabo en el aula:

- Experiencias: actividades prácticas que con su realización se pretende obtener una familiarización perceptiva con los fenómenos naturales.

- Experimentos ilustrativos: establecen una aproximación cuantitativa o cualitativa al fenómeno, permitiendo interpretar este, así como mostrar una relación entre variables o ilustrar una relación.

- Ejercicios prácticos: actividades diseñadas para aprender determinadas destrezas o procedimientos. Pueden ser para el aprendizaje de procedimientos o destrezas o para ilustrar o corroborar una teoría.

- Investigaciones: actividades dirigidas a desarrollar un problema teórico o práctico a través del diseño y la realización de un experimento, evaluando los resultados de este. Existen dos tipos principales de investigaciones: una para resolver problemas teóricos y otra para resolver problemas prácticos.

En la enseñanza de las Ciencias se abordan situaciones problema desde diversos referentes, por lo cual el propósito y alcance de la experimentación también varía, y es el maestro el encargado de guiar el proceso de enseñanza a partir de los desarrollos y habilidades que pretende favorecer en los estudiantes la construcción del conocimiento científico en situaciones cotidianas, de tal forma, que los alcances de la actividad experimental, no solo favorece la formación en un campo de conocimiento, sino las formas como la ciencia lo construye, trata de dar explicaciones y aproxima a los estudiantes a los fenómenos naturales, a partir de unas condiciones controladas que ofrecen respuestas a situaciones concretas.

Para recapitular, lo más significativo de las actividades experimentales propuestas en el aula, más que sus objetivos, radica en la posibilidad de que los niños y jóvenes avancen en sus explicaciones, por lo cual es fundamental que el maestro plantee actividades de aprendizaje a través de la experimentación, ya que permite cuestionar a los participantes y los impulsa al estudio de los fenómenos naturales, donde la experiencia personal es el vehículo donde inicia la curiosidad y desemboca en el deseo de buscar explicaciones, además de favorecer las transformación de la realidad a partir de diversas formas de abordar, comprender y tratar de dar solución a problemas cotidianos; es decir, una ciencia que no solo construye conocimiento escolar, sino que aporta en la construcción de explicaciones y es un agente transformador de la realidad.

\section{La experimentación como camino a la problematización de fenómenos concretos de la realidad}

La problematización de los fenómenos concretos de la realidad parten del deseo y de la intención de comprendery descubrir algo que fácilmente no es abordable, de la exploración de un fenómeno, de las técnicas para hacerlo accesible y del aprendizaje acerca del mismo.

Tras explicar brevemente los criterios de la problematización de los fenómenos naturales hay que centrarse en la curiosidad, ya que esta es clave en la experimentación y permite la construcción del conocimiento científico escolar.

El aprendizaje implica en la mayoría de las ocasiones el interés para que este sea significativo y transformador, además inicia en lo que es cercano a los sujetos, de mirar y observa el contexto; la curiosidad es un instinto innato que permite a los seres humanos explorar el mundo que lo rodea...posibilitando el conocimiento de su entorno y favoreciendo el aprendizaje (Pérez, 2016). En efecto, se configura como el principio del conocimiento escolar al manifestar los deseos por descubrir explicaciones a las preguntas surgidas desde tal o cual experimento; como se demostró en la investigación desarrollada por el neurocientífico Charán Ranganath: "la curiosidad prepara al cerebro para el aprendizaje y la memoria a largo plazo" (Vargas, 2018, p. 25). Tomemos el caso de un estudiante observador e inquieto por el hallazgo de respuestas sobre un tema de interés particular que podría ser-¿Por qué el agua y el aceite no se mezclan? -, esta pregunta al ser motivada por el interés del estudiante estimula la formulación de preguntas, la indagación del fenómeno particular, la observación detallada del objeto de estudio, el desarrollo de experimentos para comprobar lo que otros dicen y la explicación del fenómeno a partir de sus interpretaciones, dando apertura al aprendizaje del niño.

En suma, la curiosidad puede despertar interés científico en los estudiantes permitiéndoles comprender el mundo natural que los rodea, además de desarrollar la habilidad de argumentación y reflexión con los compañeros acerca de los fenómenos y acontecimientos naturales, dando 
como resultado aprendizajes significativos y por ende duraderos. De hecho, Alegría (2013) en su trabajo de grado de maestría en Docencia de las Ciencias Experimentales y Exactas, La exploración y experimentación del entorno natural: una estrategia didáctica para el aprendizaje de las Ciencias Naturales tiene como objetivo principal el reconocimiento, investigación y la experimentación del entorno natural como estrategia didáctica para mejorar los procesos de enseñanza-aprendizaje de las Ciencias Naturales. En esta investigación se diseñaron e implementaron guías didácticas para lograr el aprendizaje significativo de los contenidos científicos de los grados quinto y sexto grado del Colegio Limbania Velasco de Santander de Quilichao (Cauca), en el cual se concluye que los niños mostraron mayor interés y curiosidad por la temática de los líquenes, planteaban muchas preguntas, se formulaban hipótesis, tomaban muestras, clasificaban, tomaban fotos y al final de la jornada participaron en el análisis de todo lo observado, llegando a la conclusión de que los líquenes son bioindicadores, a partir del hecho de que los estudiantes pudieron observar gran variedad y cantidad de líquenes de diferentes formas, tamaños y colores, lo que les permitió concluir que al haber tantas especies de estos seres vivos en el ecosistema estudiado, los niveles de contaminación en la zona no eran tan altos, puesto que los líquenes pueden sobrevivir a este tipo de condiciones en diferentes contextos (Alegría, 2013).

Condensando lo expuesto, se puede afirmar, que la curiosidad ofrece al estudiante la posibilidad de construir esquemas de pensamiento complejos acerca de los fenómenos observados con detalle. Claro que la formulación de preguntas y la motivación que el docente desarrolle sobre las mismas contribuye en la consolidación de sus explicaciones; no obstante, la experimentación en conjunto con los dos elementos en mención es la amalgama que contribuye en la problematización de fenómenos concretos de la realidad, consolida el conocimiento científico escolar y, por tanto, el aprendizaje significativo.

Adicionalmente, en la etapa inicial de aprendizaje es indispensable motivary mediar las preguntas formuladas por el estudiante, pues esto contribuye en la comprensión del mundo que los rodea; así mismo, el cuestionamiento de las realidades es el punto de partida para avanzar en la consolidación de explicaciones y la comprensión de los fenómenos naturales. Con igual importancia, tenemos que "la experimentación y modelización de un fenómeno en el aula permite a los estudiantes construir unas primeras explicaciones, y así estos avanzan en la construcción de su conocimiento para dar explicaciones de lo que ocurre en su entorno" (Canizales, et ál., 2004, p.7). Las fallas y los errores en la experimentación no son un fracaso, sino nuevas oportunidades de reflexión y aprendizaje en el aula; son una aproximación a la comprensión del fenómeno en estudio por parte de los estudiantes y en el cual el maestro orienta dichos procesos.

Sin duda, la aplicación de estrategias y acciones en correlación con la experimentación aplicadas por el docente generan acercamiento hacia los fenómenos naturales de una forma pertinente; para reiterar, esto implica el establecimiento de la relación entre el contexto de los estudiantes y las prácticas experimentales, dado que el entorno es el lugar en donde surgen las preguntas fruto de la curiosidad del estudiante y es este el punto de partida para aproximarse al fenómeno investigado por medio de la experimentación. Así pues, "en la enseñanza de las Ciencias Naturales es necesaria la experimentación como estrategia metodológica para acceder al conocimiento; permitiendo que los estudiantes puedan entender la naturaleza de su entorno y asimilar mejor los conceptos" (Rivera, 2016, p.17); en otras palabras, existe una estrecha relación entre el contexto y las preguntas que se suscitan en los estudiantes.

Por otro lado, la confrontación de los resultados de la experimentación desarrolla en el estudiante un proceso de interpretación y construcción de nuevas explicaciones a los problemas cotidianos, que son el resultado de la curiosidad preliminar, al iniciar la búsqueda de posibles explicaciones y de la experimentación para finalizar con nuevas preguntas, que serán un elemento clave para seguir construyendo conocimiento científico en el aula.

Es necesario señalar por un lado, que el conocimiento científico escolar contribuye en la resolución de situaciones problema donde la experimentación juega un papel fundamental en la construcción de explicaciones y del surgimiento de nuevas inquietudes; la enseñanza de las Ciencias Naturales sin los problemas -entendidos como "cualquier situación prevista o espontánea que produce, por un lado, un cierto grado de incertidumbre y por el otro, una conducta tendiente a la búsqueda de su solución" (Perales, 1993, p.170) -, dejaría de ser ciencia en la medida en que se haría estática, negando su naturaleza dinámica de preguntarse y de buscar posibles soluciones a los fenómenos circundantes.

Por otra parte, se encuentra el papel fundamental de los docentes como agentes que orientan los procesos de aprendizaje de los estudiantes, quienes a su vez tienen la responsabilidad de desarrollar ejercicios práctico-experimentales que respondan a las necesidades propiciadas en su contexto; en este sentido, estos dos actores se conciben como agentes activos en la consolidación del conocimiento escolar que responda a las situaciones problémicas evidenciadas en el entorno. 
Para concluir, es necesario resaltar, que la experimentación en la escuela, especialmente en la formación inicial (básica) de los estudiantes es fundamental, debido a que se puede potenciar el aprendizaje de los niños a partir del afianzamiento de la curiosidad innata y la capacidad que tienen de asombrarse, y que al parecer se va perdiendo a medida que se avanza en los años escolares; por lo cual se convierte en una ventaja en la enseñanza de las Ciencias Naturales. El maestro puede favorecer el proceso de aprendizaje a través de trabajos experimentales cuyo adyacente sea la observación activa, las preguntas e hipótesis, la artificialización de fenómenos naturales y la búsqueda de soluciones a situaciones cotidianas y, a su vez, el desarrollo de las habilidades científicas de descripción, argumentación, análisis, apropiación y aplicación del conocimiento científico para interpelar la realidad y transformarla; por último, comprender la ciencia como un conocimiento que se construye a partir de situaciones cotidianas sin respuestas aparentes donde los estudiantes son los protagonistas en la construcción de sus nuevas explicaciones.

\section{Referencias}

Alegría, J. (2013). La exploración y experimentación del entorno natural: una estrategia didáctica para la enseñanza aprendizaje de las Ciencias Naturales. [Tesis de grado. Universidad Nacional de Colombia-Sede Palmira].

Aragón, J. (2011). La experimentación una estrategia significativa en la asignatura de Ciencias Naturales en el tercer grado. [Trabajo de Grado. Escuela Normal Urbana Federal de Cautla].

Adúriz, A., Koliopoulos, D., and Ravanis K. (2011). El "análisis del contenido conceptual" de los programas de ciencias: una posible herramienta de mediación entre la didáctica y la enseñanza de las ciencias. Enseñanza de las Ciencias, 29(3), 315-324

Baptista, P., Fernández, C., Hernández, R. (1994). Metodología de la investigación. McGraw Hill.

Caamaño, A. (2003). Experiencias, experimentos ilustrativos, ejercicios prácticos e investigación: ¿Una clasificación útil de los trabajos prácticos? Alambique, 39, 8-19.

Canizales, A., Salazar, C. y López, A. (2004). La experimentación en la enseñanza de las Ciencias Naturales en el nivel primaria. [Tesis de Maestría, Universidad Pedagógica Nacional].
Carvajal, L. (2011). Metodología de la investigación; curso general aplicado. Poemia, su casa editorial, Edición: 28.

Del Carmen, L. (2010). Las actividades prácticas en contextos multiculturales de la educación infantil y primaria. Alambique, 66, 19-27.

García, E. (2009). Historia de las ciencias en textos para la enseñanza. Editorial Universidad del Valle.

García, E. y Estany, A (2010). Filosofía de las prácticas experimentales y enseñanza de las ciencias. Revista Praxis Filosófica, 31, 7-24. https://doi. org/10.25100/pfilosofica.v0i31.3424

Hacking, I. (1996). Representar e intervenir. Paidós.

Furman, M. (2016). Educar mentes curiosas: Ia formación del pensamiento científico y tecnológico en la infancia. Santillana.

Leite, L. (2001). Razones para una utilización más fundamental del trabajo de laboratorio en la enseñanza de las Ciencias. Ministerio de Educación de Portugal.

Mora, A., y Guido, F. (2002). La enseñanza de las ciencias naturales en la escuela: problemas y perspectivas. Revista del pensamiento actual, 3(4), 17-26.

Oñate, A. (2016). La experimentación como recurso en la Educación Primaria. [Tesis de grado. Universidad Internacional de la Rioja]. https://biblioteca. unirioja.es/tfe_e/TFE001188.pdf

Ordóñez, J. y Ferreirós, J. (2002). Presentación: Hacia una filosofía de la experimentación. Revista Theoria, 17(44), 209-219.

Pedrinaci, E., Caamaño, A., Cañal, P. y De Pro, A. (2012). El desarrollo de la competencia científica: 11 ideas claves. Revista de investigación, 40 (87), 299-302.

Peña, E. (2012). Uso de actividades experimentales para recrear conocimiento científico escolar en el aula de clase, en la Institución Educativa Mayor de Yumbo. [Tesis de grado. Universidad Nacional de Colombia -Sede Palmira].

Perales, F. (1993). La resolución de problemas: una revisión estructurada. Enseñanza de las Ciencias, 11(2), 170-178.

Pérez, A. (2016). La curiosidad, el verdadero motor del aprendizaje. Nueces y neuronas, 3. 
Repositorio de Educación. (2012, 5 de abril). Repositorio de educación: http://repositorio.educacion.gov.ar:8080/dspace/ handle/123456789/99270?show=full

Rivera, A. (2016). La experimentación como estrategia para la enseñanza aprendizaje del concepto de materia y sus estados. [Tesis de Maestría. Universidad Nacional de Colombia]. http://bdigital.unal. edu.co/56352/1/24687889.2016.pdf

Rodríguez, F. (2007). Competencias comunicativas, aprendizaje y enseñanza de las Ciencias Naturales: Un enfoque lúdico. Enseñanza de las Ciencias, 6(2). 275-298.
Vargas, C. (2018). Una experiencia desde la curiosidad y el asombro, referentes para la construcción del conocimiento en la infancia. Pontificia Universidad Javeriana.

Tamayo, M. (2004). El proceso de la investigación científica: incluye evaluación y administración de proyectos de investigación. Limusa. 